\title{
ON FUZZY DIGRAPHS
}

\author{
Kishore Kumar P.K. ${ }^{1}$, S. Lavanya ${ }^{2}$ \\ ${ }^{1}$ Bharathiar University \\ Coimbatore, INDIA \\ ${ }^{2}$ Department of Mathematics \\ Bharathi Women's College \\ Chennai, INDIA
}

\begin{abstract}
In this paper we analyse some concepts of fuzzy graphs and its definitions. We discuss the concept of fuzzy digraphs, complement of fuzzy digraphs, eulerian fuzzy digraphs and tournaments in fuzzy digraphs. The concepts involving degree sequence and isomorphism in fuzzy digraphs are also discussed.
\end{abstract}

AMS Subject Classification: 05C72, 03E72

Key Words: fuzzy digraph $\mathrm{G}_{D}$, complement of fuzzy digraph $\mathrm{G}_{D}{ }^{c}$, fuzzy digraph isomorphism

\section{Introduction}

The concept of fuzzy graph evolved from the motive of uncertainty and vagueness. Set theory deals with the conditional statement of whether the element is present in the set or not denoted by 0 and 1. Fuzzy set theory deals with the concept of ambiguous situation based on the element's presence in the closed interval $[0,1]$. Lofti.A.Zadeh in [8] has found the importance of fuzziness in different environments and introduced the concept of fuzzy logic. He also analysed

Received: January 29, 2017

Revised: July 17, 2017

Published: $\quad$ July 27, 2017

(c) 2017 Academic Publications, Ltd. url: www.acadpubl.eu

${ }^{\S}$ Correspondence author 
the concept of a linguistic variable in [9] and its application to approximate reasoning-I. Later many research have been made to test the real time scenario based on fuzzy logic and its use. Several applications have been into study and useful for real life situations. Some of them are represented graphically using fuzzy digraphs. Mordeson et al. in [4] proposed the concept of fuzzy graphs and fuzzy hypergraphs. Nagoor gani et al. in his paper [6] analysed the concept of isomorphic fuzzy graphs. Lavanya et al. in their work [3] analysed the concept of fuzzy total coloring and its application to job scheduling. Eslahchi et al. in [2] identified the vertex strength of fuzzy graphs. Mourad et al. in [5] has contributed some of the ideas on isomorphic fuzzy graphs. Nirmala et al. in [7] analysed the concept of domination in fuzzy digraphs. Douglas.B.West in [1] has presented a text book on Introduction to Graph Theory with various applications. In this paper we review the concept of fuzzy graphs and also we present the brief idea of fuzzy digraphs and its classifications. Also we discuss some of the concepts involving complement, tournaments and isomorphic properties of fuzzy digraph.

\section{Main Definitions and Results}

Definition 1. (Fuzzy graph) A fuzzy graph $G=(V, \sigma, \mu)$ where $V$ is the vertex set, $\sigma$ is a fuzzy subset of $V$ and $\mu$ is a membership value on $\sigma$ such that $\mu(u, v) \leq \sigma(u) \wedge \sigma(v)$ for every $u, v \in V$.

Definition 2. (Strongly adjacent vertices) An edge is strongly adjacent if $\frac{1}{2} \min (\sigma(x), \sigma(y)) \leq \mu(x y)$.

Definition 3. (Fuzzy digraphs) A fuzzy digraph $G_{D}=\left(\sigma_{D}, \mu_{D}\right)$ is a pair of function $\sigma_{D}: \mathrm{V} \rightarrow[0,1]$ and $\mu_{D}: \mathrm{V} \times \mathrm{V} \rightarrow[0,1]$, where $\mu_{D} \leq \sigma_{D}(\mathrm{u}) \wedge \sigma_{D}(\mathrm{v})$ for $\mathrm{u}, \mathrm{v} \in \mathrm{V}$ and $\mu_{D}$ is a set of fuzzy directed edges called the fuzzy arcs.

Definition 4. (Indegree and Outdegree of a vertex in fuzzy digraphs) An Indegree of a vertex $\mathrm{u}$ in a fuzzy digraph is the sum of the $\mu_{D}$ values of the edges incident towards the vertex $\sigma_{D}(u)$. The outdegree of a vertex in a fuzzy digraph is the sum of the $\mu_{D}$ values of the edges incident from the vertex to all the other vertices. We denote the indegree by $d^{-}(u)$ and the outdegree by $d^{+}(u)$ where $u$ is any vertex in $V$.

Definition 5. (Complete fuzzy digraph) A fuzzy digraph is complete if for every pair of directed adjacent vertices $\mu_{D}(u, v)=\sigma_{D}(u) \wedge \sigma_{D}(v)$.

Definition 6. (Fuzzy regular digraph) A fuzzy digraph is said to be fuzzy regular digraph if every node has the same indegree and outdegree as every 
other node.

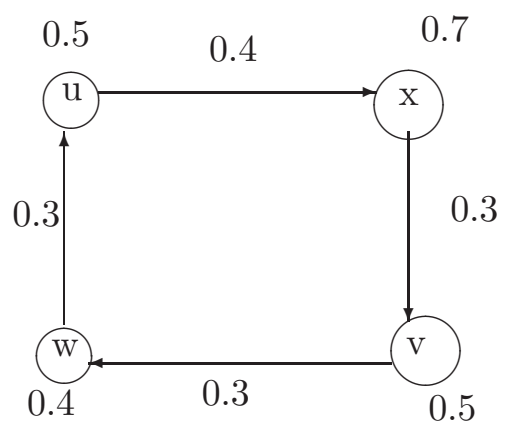

Fuzzy Digraph

Definition 7. ( $k$-fuzzy coloring of $G=(X, \sigma, \mu))$ The properties which a fuzzy coloring should satisfy are below:

(a) $\vee \Gamma=\sigma ;$

(b) $\gamma_{i} \wedge \gamma_{j}=0$

(c) For every strong edge $x y$ of $G, \min \left\{\gamma_{i}(x), \gamma_{i}(y)\right\}=0(1 \leq i \leq k)$.

The minimum of all the indegree vertices of the fuzzy digraph is denoted by $\delta^{-}(\mathrm{G})$ and the maximum of all the outdegree vertices of the fuzzy digraph is denoted by $\delta^{+}(\mathrm{G})$.

Theorem 8. If $G_{D}$ is a fuzzy digraph with $n \geq 3$ vertices and $\delta^{+}(G) \geq 0$, $\delta^{-}(G) \geq 0$, then $G$ contains a cycle.

Proof. Let $G_{D}$ be a fuzzy digraph with $\mathrm{n} \geq 3$ vertices with $\delta^{+}(\mathrm{G})>0$ and $\delta^{-}(\mathrm{G})>0$. Suppose that $G_{D}$ does not contains a cycle. Let $P$ be a maximal path in $G_{D}$. Let $z$ be a last vertex of $P$. because $P$ cannot be extended, there is a predecessor for the vertex $z$. Let $u$ be the predecessor of $z$. since $G$ does not contain a cycle the vertex $u$ may not contain an indegree which is a contradiction to the fact that $\delta^{+}(\mathrm{G})>0$ and $\delta^{-}(G)>0$. Hence we have, $\delta^{+}(G)$ $>0$ and $\delta^{-}(G)>0$ and this contradiction occurs on the assumption that $G_{D}$ does not contain a cycle. Hence $G_{D}$ contains a cycle.

Definition 9. A fuzzy digraph is eulerian if and only if $d^{+}(\mathrm{v})=d^{-}$(v)for each vertex $v$ and the underlying graph has atmost one trivial component. 


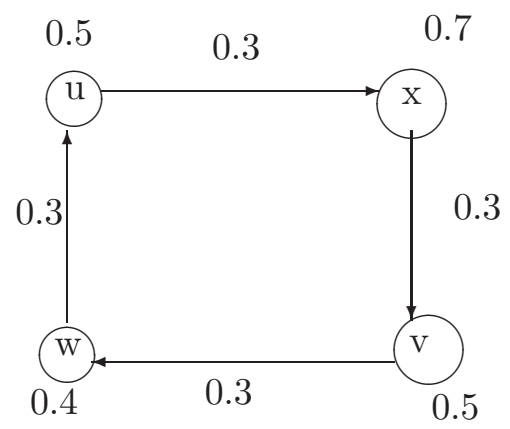

\section{Fuzzy Eulerian Digraph}

Theorem 10. A fuzzy digraph is eulerian iff $d^{+}(\mathrm{v})=d^{-}(\mathrm{v})$ and all the vertices are strongly connected.

Proof. Let $G_{D}$ be a fuzzy digraph which is eulerian.Every edges can be traversed only if the number of edges incident to all vertices have same indegree and outdegree. Hence we have $d^{+}(v)=d^{-}(v)$ for some vertex $v$ in $V$. On the other hand if $d^{+}(v)=d^{-}(v)$ and all the vertices are strongly connected. i.e., $\frac{1}{2} \min \left(\sigma_{D}(u), \sigma_{D}(v)\right) \leq \mu_{D}(u v)$, then there exist a path from all the vertices to each other. Also their indegree and outdegree are same. Therefore, $G_{D}$ is eulerian.

Definition 11. (Oriented fuzzy digraph) An orientation of a fuzzy digraph $G$ is a fuzzy digraph $G_{D}$ obtained from $\mathrm{G}$ by choosing an orientation from $x$ to $y$ or $y$ to $x$ for each edge $x y \in E\left(G_{D}\right)$. An oriented graph is an orientation of a simple graph.

Definition 12. (Tournament of a fuzzy digraph) A tournament is an orientation of a complete fuzzy graph.

Definition 13. In a fuzzy digraph a queen is a vertex from which every vertex is reachable by a path of length atmost 2 .

Theorem 14. Every tournament has a queen in a fuzzy digraph if all its edges are strongly connected.

Proof. Let $u$ be a vertex in a tournament $T$ (say). If $u$ is not a queen then any other vertex $v$ is not reachable from $u$ by path of length atmost 2 . Also the edges in $G_{D}$ are not strongly connected. Hence no successor of $u$ is a predecessor of $v$. Because $T$ is an orientation of fuzzy clique, every successor of $u$ must therefore be a successor of $y$. Also we have $v \rightarrow u$. Because $d^{+}(v)$ $>d^{+}(u)$. If we proceed with the vertex $v$ which is not a queen, repeating the 
process to find another vertex $z$ with larger outdegree. Because $\mathrm{T}$ is finite it is not possible to obtain the vertices of successively higher outdegree. Because, if we find a queen then the process is terminated. The below shows all the edges are strong arc and hence can be traversed from any vertex having a path of length atmost 2 .

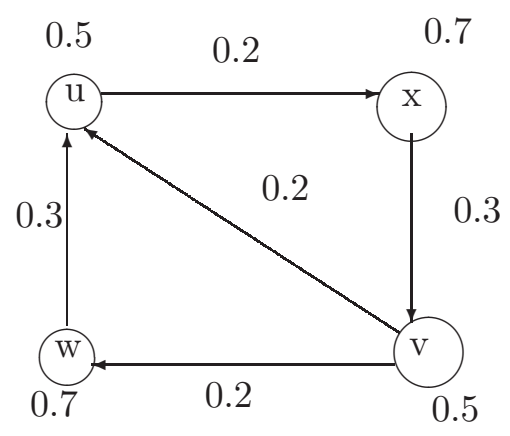

$G_{D}$ with strong arcs

The below graph shows a fuzzy digraph with an arc to be weak. Hence it is not possible to traverse through the weak arc. In this digraph the arc $x v$ is weak and hence we cannot traverse from $u$ to $v$ through the vertex $x$.

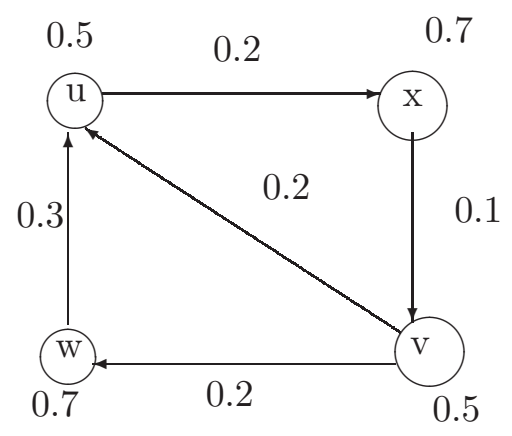

$G_{D}$ with strong and weak arcs

Definition 15. (Complement of a fuzzy digraph) The complement of a fuzzy digraph is the fuzzy digraph $G_{D}$ with same membership values of vertices in $V$ and the edges $E$ adhering to the condition $\min (\sigma(u), \sigma(v))-\mu(u v)$ for all the vertices $u, v \in V$.i.e., $\mu_{D}(u v)=\sigma_{D}(u) \wedge \sigma_{D}(v)$ for some $u, v$ in $V$. 


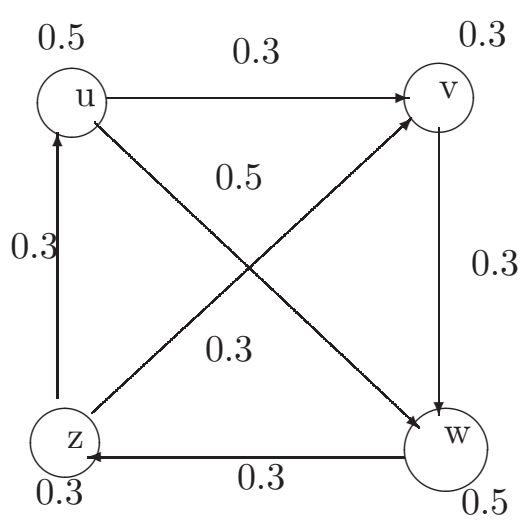

$G_{D}$
0.5

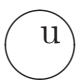

0.3
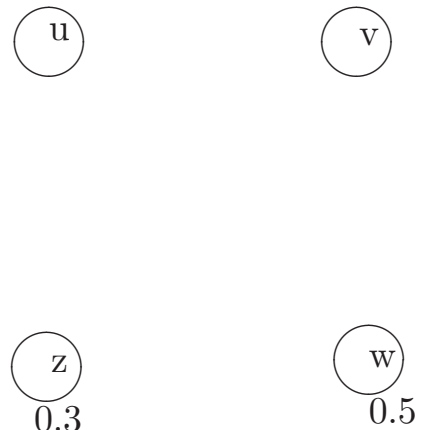

Theorem 16. In any complement of a complete fuzzy digraph the indegree and outdegree of the vertices are always zero.

Proof. Let $G_{D}$ be a fuzzy digraph which is complete with $n$ indegree and outdegree(say). Then $d^{+}(u)=d^{-}(v)=n$. Let $G_{D}^{c}$ be the complement of the fuzzy digraph. Then by definition of complement, $d^{+}(u)=d^{-}(v)=0$ in complement of fuzzy digraph. Suppose if $G_{D}$ is not complete, then any pair of vertices may not be adjacent to each other. i.e., $\mu_{D}(u v)<\sigma_{D}(u) \wedge \sigma_{D}(v)$. Hence there exist an arc $u v$ in $G_{D}^{c}$ such that $u$ and $v$ are adjacent. This result is a contradiction to the fact that $G_{D}$ is not complete. Hence $G_{D}$ should be complete to have zero degree in its complement. Hence $d^{+}(u)=d^{-}(v)=0$ in any fuzzy complete digraph.

\section{Isomorphic properties of fuzzy digraphs}

Definition 17. (Homomorphism of fuzzy digraphs) A homomorphism of fuzzy digraphs $\phi: G_{1 D} \rightarrow G_{2 D}$ is a map $\phi: V_{1} \rightarrow V_{2}$ satisfying the condition $\sigma_{D}(\mathrm{x}) \leq \sigma_{D}(\phi(\mathrm{x}))$ and $\mu_{1 D}(\mathrm{xy}) \leq \mu_{2 D}(\phi(\mathrm{x}), \phi(\mathrm{y}))$ for all $\mathrm{x}, \mathrm{y}$ in $\mathrm{V}$.

Definition 18. (Isomorphism of fuzzy digraphs) Let $G_{1 D}:\left(\sigma_{1 D}, \mu_{1 D}\right)$ and $G_{2 D}:\left(\sigma_{2 D}, \mu_{2 D}\right)$ be two fuzzy graphs with $\sigma_{1 D}=V_{1}$ and $\sigma_{2 D}=V_{2}$.

An isomorphishm between two fuzzy digraphs $G_{1 D}$ and $G_{2 D}$ is a bijective map $\phi: V_{1} \rightarrow V_{2}$ that satisfy $\sigma_{1 D}=\sigma_{2 D}(\phi(x))$ for all $x$ in $V$ and $\mu_{1 D}(x y)=$ $\mu_{2 D}(\phi(x), \phi(y))$, for all $x, y$ in $V$. We have $G_{1 D} \cong G_{2 D}$.

An automorphism of $G_{D}$ is an isomorphism of $G_{D}$ with itself. 
Definition 19. (Degree sequence of fuzzy digraphs) The degree sequence of fuzzy digraphs is the indegree and outdegree of all the vertices of the fuzzy digraph arranged in ascending or descending order from 0 to 1 or 1 to 0 .

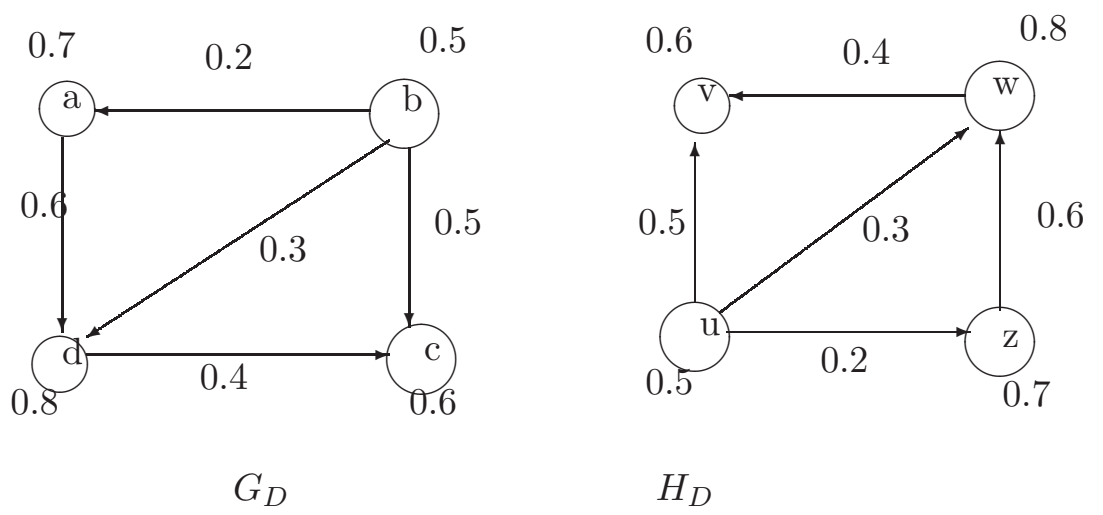

In the above fuzzy digraphs $G_{D}$ and $H_{D}$ we analyse the number of edges and vertices to be 5 and 4 respectively. One can verify the indegree and outdegree of each vertex of the fuzzy digraphs. Also the indegree sequence of the graph $G_{D}$ is $0,0.2,0.9,0.9$. Also the outdegree sequence of $G_{D}$ is $0,0.4,0.6,1$. Similarly the indegree sequence of the graph $H_{D}$ is $0,0.2,0.9,0.9$ and the outdegree sequence of the graph $H_{D}$ is $0,0.4,0.6,1$ respectively. We know that a fuzzy digraph is isomorphic if there is a one to one correspondence between the vertices and edges having same indegree and outdegree. The functional mapping from $G_{D}$ to $H_{D}$ are $a=f(z), c=f(v), b=f(u), d=f(w)$ which forms an isomorphic relation between $G_{D}$ and $H_{D}$. One can find more isomorphic relations with different mappings of the vertices of $G_{D}$ and $H_{D}$.

\section{Conclusion and Future Work}

Thus we have discussed the concept on fuzzy graphs. Introduced the notion of fuzzy digraphs with different examples. Discussed some of the properties on complement of fuzzy digraphs, fuzzy eulerian digraphs, tournament on fuzzy digraphs. Also we analysed the ideas on isomorphic properties of fuzzy digraphs based on its degree sequence and bijective mapping. In future we can get an extension on more of its properties in fuzzy digraphs and work on its real time applications. 


\section{References}

[1] Douglas B. West, Introduction to Graph Theory, Text book in Eastern Economy Edition, University of Illinois, (2014).

[2] Ch. Eslahchi, B. N. Onagh, Vertex strength of fuzzy graph, Hindawi Publishing Corporation, 2006 (1998), 1-9.

[3] S. Lavanya and R. Sattanathan, Fuzzy total coloring of fuzzy graphs, International Journal of Information Technology and Knowledge Management, 2 (1998), 37-39.

[4] J. N. Mordeson and C. S. Peng, Operations on fuzzy graphs, Information Sciences, 79 (1994), 159-170.

[5] Mourad Oqla Massa'deh and Abd Al Karim Baareh, Some contribution on Isomorphic fuzzy graphs, Advances and Applications in Discrete Mathematics, 11 (2013), 199 - 206.

[6] A. Nagoor Gani and J. Malarvizhi, Isomorphism on fuzzy graphs, International Journal of Computational and Mathematical Sciences, 2 (2008), 200-206.

[7] G. Nirmala and M. Sheela, Domination in Fuzzy Digraphs, Aryabhatta Journal of Mathematics and informatics, 5 (2013), 275 - 278.

[8] L. A. Zadeh, Fuzzy sets, Information and Control, 8 (1965), 338-353.

[9] L. A. Zadeh, The concept of a linguistic variable and its application to approximate reasoning-I, Information Sciences, 8 (1975), 199-249. 